\title{
A review of population-based prevalence studies of physical activity in adults in the Asia-Pacific region
}

Rona Macniven ${ }^{1 *}$, Adrian Bauman ${ }^{1}$ and Marian Abouzeid ${ }^{1,2}$

\begin{abstract}
Background: Physical activity (PA) surveillance is an important component of non-communicable disease risk factor monitoring, and occurs through national and international surveillance systems. This review identifies population PA estimates for adults in the Asia-Pacific region, and examines variation in trends and prevalence rates obtained using different PA measures.

Methods: Data were obtained from a MEDLINE search; World Health Organization's Global Health Infobase; Government websites and reference lists of relevant papers. Inclusion criteria included: national studies or those reporting large scale population-level data; data published from 2000 to 2010 and trend data prior; sample sizes over $n=1000$, or fewer subjects in small nations.

Results: In total, 56 population surveys from 29 Asia-Pacific countries were identified. Data on 'sufficient physical activity' amongst adults were available from 45 studies (80\%), with estimates ranging from 7\% to 93\% (median $62 \%$, inter-quartile range $40 \%-85 \%$ ). For 14 countries, estimates of 'sufficient activity' were documented in multiple surveys using different methods, with the largest variation from 18\% to 92\% in Nepal. Median or mean METminutes/day, reported in 20 studies, ranged from 6 to 1356. Serial trend data were available for 11 countries (22\%), for periods spanning 2-10 years. Of these, five countries demonstrated increases in physical activity over time, four demonstrated decreases and three showed no changes.

Conclusions: Many countries in the Asia-Pacific region collect population-level PA data. This review highlights differences in estimates within and between countries. Some differences may be real, others due to variation in the PA questions asked and survey methods used. Use of standardized protocols and measures, and combined reporting of data are essential goals of improved international PA surveillance.
\end{abstract}

\section{Background}

Regular physical activity is an important aspect of noncommunicable disease (NCD) prevention $[1,2]$ and inactivity is the fourth largest contributor to global mortality and morbidity [3-5]. Physical activity is undertaken in several domains of daily life, including work, transport and leisure time [6]. This distinction is especially important in developing and transitional countries, where recreational physical activity makes a smaller contribution to total energy expenditure than occupational or transportation related activity $[7,8]$. The measurement of

\footnotetext{
* Correspondence: rona.macniven@sydney.edu.au

'Prevention Research Collaboration, School of Public Health, University of Sydney, Sydney, Australia

Full list of author information is available at the end of the article
}

population levels of physical activity is necessary to guide health promotion initiatives and policy formulation and to assess the impact of large-scale policies and programs designed to increase activity [9]. For adults, physical activity is usually assessed by self-report, through interviews or self-administered questionnaires. The concept of 'sufficient' activity relates to the proportion of the population achieving recommended levels of physical activity for promoting health and preventing disease [10].

In countries such as the United States, Finland, and Canada, standardized population-level physical activity surveillance in adults has occurred for several decades [11-13]. In other countries, surveillance systems have been less consistent and have asked different physical

\section{Ciomed Central}


activity questions and are therefore not comparable [12]. Physical activity instruments vary with regard to the questions asked; recall periods and the areas of daily life assessed [9]. Many surveys in developed countries explore leisure time activities only. These may yield very different prevalence estimates compared to newer international questionnaires that examine a wider range of domains for expending energy, including work, travel, recreation and domestic settings. Recent international measures have included estimates of total physical activity and have asked about the different domains in which adults can report activity. The most common measures are the International Physical Activity Questionnaire (IPAQ) and the Global Physical Activity Questionnaire (GPAQ) $[14,15]$. The short-form of IPAQ considers all domains collectively in generic questions, and has been used to compare prevalence rates within and between countries worldwide [16,17]. Given the length of the long form of IPAQ, its use for international comparisons has been less frequent. The intermediate-length Global Physical Activity Questionnaire (GPAQ) [15] was developed by the World Health Organization (WHO) to measure physical activity across the work, transport and recreation domains separately, and is used in many countries as part of the WHO STEPwise approach to Non-Communicable Disease surveillance (STEPS) [18]. Both IPAQ and GPAQ are reliable and valid measures $[14,15,19]$. Moreover, both IPAQ-long and GPAQ are suitable for use in developing countries where physical activity is more likely to be accrued across a range of domains $[7,8]$.

The Asia-Pacific region comprises diverse, predominantly low-middle income countries (LMIC) [20] ranging from large and densely populated Asian countries to tiny Pacific island nations. Given the major increases in NCDs in the region [21,22], risk factor surveillance, including physical activity, is essential.

This paper has two purposes: i) to identify and compare published national physical activity prevalence estimates in adults aged 18-64 years in the Asia-Pacific countries in the WHO Western Pacific (WPRO) and South East Asian (SEARO) regions; ii) to examine variations in the prevalence and trends in physical activity within and between these countries, using different surveillance instruments.

\section{Methods}

Data on physical activity prevalence across the AsiaPacific region was obtained from several sources. A MEDLINE search used a combination of search terms including 'physical activity' or 'inactivity' or 'exercise', and 'prevalence', Asia, Pacific, Southeast Asia, Central Asia and individual country names. The full search strategy can be found in Additional file 1. Additional searching was carried out through the Asia-Pacific Physical Activity Network (AP-PAN) [23]; resources identified through AP-PAN and WHO links in the region included country-level health and NCD reports. Reports and websites of governmental and non-governmental organisations were also reviewed. This comprises a large amount of grey literature, not cited in indexed academic databases; however, this method generated many of the estimates used.

Relevant data were also extracted from sources including the World Health Organization's Global Health Infobase (STEPS) [18], the World Health Survey [16] the International Prevalence Study [17]. In some cases, authors were contacted for further information and raw data. Additional studies known to authors or AP-PAN members were obtained. Reference lists of retrieved studies were also reviewed.

Published literature and electronic resources accessed were limited to those in the English language. In order to profile recent epidemiology, only studies conducted between 2000 and 2010 were included. Where possible studies with sample sizes over 1000 were sought; however in some cases, nations with small populations that had conducted studies with fewer subjects were included. Where serial surveys using the same methodology were available, data prior to 2000 was included to establish physical activity trends. Where more than one survey was available for a country, all relevant data were included. This review was limited to nationally representative population-level studies or those reporting large-scale, sub-national population-level data from specific region(s). Studies involving children or adolescents or adults aged 65 years and above were excluded.

Physical activity estimates are presented as described in the original source documentation. For surveys where data were reported separately for males and females, the mean of these values was used as a population proxy estimate, as both genders tended to be proportionally represented or were weighted proportionally in samples. Some studies reported the percent achieving a threshold level 'sufficiently active' for health, defined as achieving 'at least $150 \mathrm{~min}$ of moderate-intensity aerobic physical activity throughout the week' [10]. Other studies reported mean or median MET-minutes per day of physical activity. MET-minutes are metabolic equivalents that describe the intensity of physical activity (amount of energy expended) relative to sitting quietly [24]. Where applicable, data obtained from other measures unique to individual surveys were also included.

\section{Results}

A total of 56 surveys from 29 countries in the Asia-Pacific region were included in this review. Sample sizes ranged from 586 in the small Pacific island nation of 
Tokelau to 42,500 in India. Nationally representative samples were used in 37 studies (66\%). Table 1 shows the physical activity prevalence data by country and outlines year of survey, sample size, representativeness and the domains assessed. A list of 12 countries where national or representative data was unavailable or unknown for the period 2000-2010 can be found in Additional file 2.

Nineteen surveys reported data on median MET-minutes/day, with estimates ranging from 98 in Vietnam to 1461 in India. Six surveys contained information on mean MET-minutes, ranging from 6 in China to 161 in Bangladesh. Additionally, in Indonesia, median METminutes/day were reported according to domain of physical activity (work: $60 \mathrm{MET}$-minutes; transport: 26 MET-minutes; leisure: 17 MET-minutes) and three countries reported mean MET-minutes by domain. In the Marshall Islands, the travel domain contributed 45 MET-minutes compared with work (18 MET-mins) and leisure (12 MET-mins) domains. In Kiribati and Samoa, the work domain contributed most (39 and 9 MET-minutes respectively) to the total, followed by travel ( 25 and 3 MET-minutes respectively) and leisure (5 and 2 METminutes respectively) domains.

Surveys other than the IPAQ or GPAQ were reported from nine countries. Six of these countries used multiple additional survey instruments, totalling 18 studies using other methodologies. Nine of these surveys $(16 \%$ of the total sample) were national health surveys, three (5\%) were national sport surveys and the remaining six (11\%) used unique measurement tools. Ten surveys measured the percent of sufficiently active adults, with estimates varying from 14\% in Singapore to $70 \%$ in New Zealand.

Comparisons can be made among surveys that used the same measures. Nineteen of the surveys (34\%) used the IPAQ, as part of the World Health Survey. Estimates of the proportion 'sufficiently active' ranged from 54\% in Malaysia to $93 \%$ in the Philippines (median 90\%, interquartile range $80-92 \%$ ). Median MET-minutes of activity varied from 98 in Vietnam to 1461 in India (median 694 MET-minutes, inter-quartile range 143-1156).

Eighteen surveys (37\%) used the GPAQ, as part of the WHO NCD STEPS Surveys. Fifteen of these provided estimates of the percent 'sufficiently active', ranging from $7 \%$ in Maldives to $89 \%$ in Mongolia (median 53.5\% inter-quartile range 44.5-80.5). Ten surveys provided median MET-minutes, ranging from 134 in Indonesia to 918 in Bangladesh and Myanmar and five provided mean MET-minutes ranging from 69 in Kiribati to 111 mean MET-minutes in Tokelau.

Eighteen surveys provided data on both percent 'sufficiently active', and median and/or mean MET-minutes of physical activity. For the majority of these surveys, both measures were similar. For example, in the
Philippines where 93\% were 'sufficiently active', the median activity was $1158 \mathrm{MET}$-minutes/day. However, in Vietnam, the percent 'sufficiently active' was high at $92 \%$ but the median activity was discordant at only 98 MET-minutes.

Of the 56 surveys reported here, 11 (22\%) were conducted more than once providing trend information. Of these, five countries (Indonesia, New Zealand, Singapore, Taiwan, and Thailand) demonstrated increases in physical activity levels over time. Three surveys conducted in Australia, the Republic of Korea and Japan demonstrated decreases in physical activity levels over time. The remaining three from Australia, Japan and New Zealand did not show significant changes in either direction over time (data not shown).

Figure 1 displays estimates from the 45 surveys (80\%) providing data on "sufficiently active" adults in the population, ranging from $7 \%$ in the Maldives to $93 \%$ in the Philippines (median estimate 62\%, inter-quartile range 40-85). In 14 countries more than one survey has been used. Six such nations reported prevalence data from both IPAQ and GPAQ and in all these cases, higher proportions of 'sufficient activity' were reported using the IPAQ measure compared with the GPAQ. The greatest variation was in Nepal; with estimates of $18 \%$ sufficiently active obtained using GPAQ, compared with an estimate of $92 \%$ from IPAQ.

Surveys that used other instruments to examine leisure-time physical activity reported substantially lower prevalence estimates compared with countries using the multi-domain measures of IPAQ and/or GPAQ. Similar differences in point estimates derived from IPAQ and GPAQ are evident for median MET-minutes, with three of the four countries with data from both surveys reporting higher values using IPAQ than GPAQ. This discrepancy was most pronounced in India, with estimates varying from 1461 median MET-minutes from IPAQ to 356 median MET-minutes from GPAQ. Only Myanmar reports a higher median MET-minutes value using GPAQ than IPAQ (918 compared with 694).

\section{Discussion}

This paper is a review of published population-level physical activity estimates among adults in the AsiaPacific region. Standardised and comparative physical activity prevalence information from other regions currently exists, such as the IPAQ survey conducted in 51 countries through the 2002 World Heath Survey [16] and the 20-country International Prevalence Study [17]. Whilst these studies give estimates of physical activity prevalence in countries using the same measure and similar protocols, unexpected results in some countries are apparent. Our paper is the first to report prevalence data from many countries that have collected 
Table 1 Prevalence of physical activity in the Asia-Pacific region

\begin{tabular}{|c|c|c|c|c|c|c|c|c|c|c|}
\hline Country & Survey & $\begin{array}{l}\text { Year } \\
\text { (s) }\end{array}$ & $\begin{array}{l}\text { Sample } \\
\text { size }\end{array}$ & Scope & $\begin{array}{l}\text { Population } \\
\text { Weighting }\end{array}$ & Domain & $\begin{array}{l}\% \\
\text { sufficiently } \\
\text { active }\end{array}$ & $\begin{array}{l}\text { Median- } \\
\text { or mean } \\
\text { METmins/ } \\
\text { day }\end{array}$ & Other measures & Trends \\
\hline \multirow[t]{8}{*}{ 2. Australia } & Active Australia & 1997 & 1997: 6803 & National & Yes & $L$ & 1997: 62 & N/A & N/A & (1) \\
\hline & Survey [25] & & & & & & & & & $\begin{array}{l}\downarrow \text { over } 3 \\
\text { yrs }\end{array}$ \\
\hline & & 1999 & 1999: 3841 & & & & 1999: 57 & & & \\
\hline & & 2000 & 2000: 3590 & & & & 2000: 58 & & & \\
\hline & $\begin{array}{l}\text { National Health } \\
\text { Survey [26-28] }\end{array}$ & 1995 & $\begin{array}{l}1995: \\
39110\end{array}$ & & Yes & $L$ & 1995-6: 39 & & & $\begin{array}{l}\text { (2) } \leftrightarrow \\
\text { over } 10 \\
\text { yrs }\end{array}$ \\
\hline & & 2000 & $\begin{array}{l}2000: \\
17918 \\
\end{array}$ & & & & 2000: 40 & & & \\
\hline & & $\begin{array}{l}2004- \\
5 \\
\end{array}$ & $\begin{array}{l}\text { 2004/5: } \\
19501\end{array}$ & & & & 2004-5: 39 & & & \\
\hline & $\begin{array}{l}\text { IPAQ Short Form } \\
{[17]}\end{array}$ & 2003 & 2691 & & Yes & all & 83 & & & (3) $N / A$ \\
\hline \multirow[t]{2}{*}{$\begin{array}{l}3 . \\
\text { Bangladesh }\end{array}$} & $\begin{array}{l}\text { WHO NCD STEPS } \\
\text { Survey (GPAQ) [29] }\end{array}$ & 2002 & 11409 & $\begin{array}{l}\text { Sub- } \\
\text { national }\end{array}$ & Yes & W, T & $\mathrm{N} / \mathrm{A}$ & 161 mean & $\mathrm{N} / \mathrm{A}$ & $\mathrm{N} / \mathrm{A}$ \\
\hline & $\begin{array}{l}\text { World Health Survey } \\
\text { (IPAQ) [16] }\end{array}$ & 2003 & 5166 & National & Yes & all & & $\begin{array}{l}131 \\
\text { median }\end{array}$ & & \\
\hline \multirow[t]{2}{*}{$\begin{array}{l}4 . \\
\text { Cambodia }\end{array}$} & $\begin{array}{l}\text { Diabetes and } \\
\text { associated disorders } \\
\text { in Cambodia [30] }\end{array}$ & 2004 & $\begin{array}{l}\text { (1) } 1195 \\
\text { (Kampong } \\
\text { Cham } \\
\text { region) }\end{array}$ & $\begin{array}{l}\text { Sub- } \\
\text { national }\end{array}$ & unknown & $W, T, L$ & (1) 51 & $\mathrm{~N} / \mathrm{A}$ & $\mathrm{N} / \mathrm{A}$ & $N / A$ \\
\hline & & & $\begin{array}{l}\text { (2) } 1051 \\
\text { (Siem } \\
\text { Reap } \\
\text { region) }\end{array}$ & & & & (2) 68 & & & \\
\hline \multirow[t]{6}{*}{ 5. China } & InterASIA [31] & $\begin{array}{l}2000- \\
1\end{array}$ & 15540 & National & Unknown & W, L & 66 & N/A & (1) N/A & N/A \\
\hline & $\begin{array}{l}\text { World Health Survey } \\
\text { (IPAQ) [16] }\end{array}$ & 2002 & 3596 & $\begin{array}{l}\text { Sub- } \\
\text { national }\end{array}$ & Yes & all & 90 & $\begin{array}{l}697 \\
\text { median }\end{array}$ & N/A & \\
\hline & $\begin{array}{l}\text { Cardiovascular } \\
\text { Health of residents: } \\
\text { cities in China [32] }\end{array}$ & 2001 & 2165 & $\begin{array}{l}\text { Sub- } \\
\text { national }\end{array}$ & Unknown & L & N/A & 6 mean & N/A & \\
\hline & $\begin{array}{l}\text { Chinese Third } \\
\text { National Health } \\
\text { Services Survey [33] }\end{array}$ & 2003 & 19057 & National & No & $L$ & $\mathrm{~N} / \mathrm{A}$ & N/A & $\begin{array}{l}\text { Regularly } \\
\text { exercising: } 13.6 \%\end{array}$ & \\
\hline & $\begin{array}{l}\text { IPAQ Short Form } \\
{[17]}\end{array}$ & 2002 & $\begin{array}{l}1593 \\
\text { (Shanghai) }\end{array}$ & $\begin{array}{l}\text { Sub- } \\
\text { national }\end{array}$ & Yes & all & 93 & N/A & N/A & \\
\hline & $\begin{array}{l}\text { IPAQ Short Form } \\
{[17]}\end{array}$ & $\begin{array}{l}2002- \\
3\end{array}$ & $\begin{array}{l}4886 \text { (SAR } \\
\text { Hong } \\
\text { Kong) } \\
\end{array}$ & $\begin{array}{l}\text { Sub- } \\
\text { national }\end{array}$ & Yes & all & 85 & N/A & $\mathrm{N} / \mathrm{A}$ & \\
\hline \multirow[t]{3}{*}{ 6. Fiji } & $\begin{array}{l}\text { WHO NCD STEPS } \\
\text { Survey (adapted } \\
\text { from GPAQ) [34] }\end{array}$ & 2002 & 6,783 & National & Yes & $W, T, L$ & $\mathrm{~N} / \mathrm{A}$ & N/A & $\begin{array}{l}\text { Mod-high active } \\
\text { W: } 49.5 \%\end{array}$ & $\mathrm{~N} / \mathrm{A}$ \\
\hline & & & & & & & & & T: $85.1 \%$ & \\
\hline & & & & & & & & & L: $24.0 \%$ & \\
\hline \multirow[t]{3}{*}{ 7. India } & $\begin{array}{l}\text { WHO modified } \\
\text { STEPS Survey (GPAQ) } \\
{[35]}\end{array}$ & $\begin{array}{l}2003- \\
5\end{array}$ & 42500 & $\begin{array}{l}\text { Sub- } \\
\text { national }\end{array}$ & Yes & $W, T, L$ & 84 & $\begin{array}{l}\text { (1) } 356 \\
\text { median }\end{array}$ & N/A & N/A \\
\hline & $\begin{array}{l}\text { World Health Survey } \\
\text { (IPAQ) [16] }\end{array}$ & 2003 & 7945 & & $\overline{Y e s}$ & all & 88 & $\begin{array}{l}1461 \\
\text { median }\end{array}$ & & \\
\hline & $\begin{array}{l}\text { IPAQ Short Form } \\
{[17]}\end{array}$ & 2003 & 1005 & & Yes & all & 77 & N/A & & \\
\hline
\end{tabular}


Table 1 Prevalence of physical activity in the Asia-Pacific region (Continued)

\begin{tabular}{|c|c|c|c|c|c|c|c|c|c|c|}
\hline \multirow[t]{4}{*}{ 8. Indonesia } & $\begin{array}{l}\text { Indonesia Health } \\
\text { Survey } 2001 \text { [36] }\end{array}$ & 2001 & 13131 & National & Yes & $W, T, L$ & 36 & $\mathrm{~N} / \mathrm{A}$ & $N / A$ & $N / A$ \\
\hline & $\begin{array}{l}\text { WHO STEPS survey } \\
\text { (GPAQ) }[37,38]\end{array}$ & 2003 & 2003: 1855 & $\begin{array}{l}\text { Sub- } \\
\text { national }\end{array}$ & Yes & $W, T, L$ & $\begin{array}{l}\text { 2003: } 67 \\
\text { 2006: } 78\end{array}$ & $\begin{array}{l}\text { 2003: } 134 \\
\text { mean }\end{array}$ & & $\begin{array}{l}\text { pover } 3 \\
\text { yrs }\end{array}$ \\
\hline & & 2006 & 2006: 1927 & & & & & $\begin{array}{l}\text { 2006: W: } \\
60\end{array}$ & & \\
\hline & & & & & & & & $\begin{array}{l}\text { T: } 26, \mathrm{~L}: 17 \\
\text { median }\end{array}$ & & \\
\hline \multirow[t]{18}{*}{ 9. Japan } & $\begin{array}{l}\text { National Sport Life } \\
\text { Survey [39-42] }\end{array}$ & 2000 & 2000: 2238 & National & unknown & L & N/A & N/A & $\begin{array}{l}\text { "Active Sport } \\
\text { Participant" (> 2x } \\
\text { wk, > } 30 \text { min, > } \\
\text { moderate) }\end{array}$ & $\begin{array}{l}\downarrow \text { over } 2 \\
\text { yrs } \\
\text { then } \\
\text { 个over 2 }\end{array}$ \\
\hline & & 2002 & 2002: 2267 & & & & & & & \\
\hline & & 2004 & 2004: 2288 & & & & & & & \\
\hline & & 2006 & 2006: 1867 & & & & & & & \\
\hline & & & & & & & & & 2000: 18\% & \\
\hline & & & & & & & & & 2002: 13\% & \\
\hline & & & & & & & & & 2004: 16\% & \\
\hline & & & & & & & & & 2006: 16\% & \\
\hline & $\begin{array}{l}\text { National Health and } \\
\text { Nutrition Survey, } \\
\text { Japan [18] }\end{array}$ & 2000 & 2000: 6815 & National & Unknown & unknown & N/A & & $\begin{array}{l}\text { "Inactive" }(<30 \\
\text { min, }<2 \times \text { wk in } \\
\text { last yr) }\end{array}$ & $\begin{array}{l}\leftrightarrow \text { over } \\
3 \text { yrs }\end{array}$ \\
\hline & & 2001 & $\begin{array}{l}\text { 2001: } \\
\text { unknown }\end{array}$ & & & & & & & \\
\hline & & 2002 & 2002: 9723 & & & & & & & \\
\hline & & 2003 & 2003: 9214 & & & & & & 2000: 71\% & \\
\hline & & & & & & & & & 2001: 72\% & \\
\hline & & & & & & & & & 2002: 70\% & \\
\hline & & & & & & & & & 2003: 77\% & \\
\hline & $\begin{array}{l}\text { National Health and } \\
\text { Nutrition Survey, } \\
\text { Japan [18] }\end{array}$ & 2004 & 2004: 4573 & National & Unknown & unknown & 21 & & $\mathrm{~N} / \mathrm{A}$ & $\mathrm{N} / \mathrm{A}$ \\
\hline & $\begin{array}{l}\text { Fifth National Survey } \\
\text { of CVD [18] }\end{array}$ & 2000 & 8369 & National & Unknown & unknown & $N / A$ & & $\begin{array}{l}<4000 \text { steps/day: } \\
22 \%\end{array}$ & $\mathrm{~N} / \mathrm{A}$ \\
\hline & $\begin{array}{l}\text { IPAQ Short Form } \\
{[17]}\end{array}$ & 2003 & 4959 & $\begin{array}{l}\text { Sub- } \\
\text { national }\end{array}$ & Yes & all & 57 & & $\mathrm{~N} / \mathrm{A}$ & N/A \\
\hline \multirow[t]{4}{*}{ Kiribati } & $\begin{array}{l}\text { WHO STEPS survey } \\
\text { (GPAQ) [43] }\end{array}$ & $\begin{array}{l}2004- \\
6\end{array}$ & 1,288 & National & Yes & $W, T, L$ & 50 & 69 mean & $\mathrm{N} / \mathrm{A}$ & $\mathrm{N} / \mathrm{A}$ \\
\hline & & & & & & & & W: 39 & & \\
\hline & & & & & & & & $\mathrm{T}: 25$ & & \\
\hline & & & & & & & & L: 5 & & \\
\hline \multirow[t]{2}{*}{$\begin{array}{l}\text { 10. Republic } \\
\text { of Korea }\end{array}$} & $\begin{array}{l}\text { National Health \& } \\
\text { Nutrition Survey } \\
{[44,45]}\end{array}$ & $\begin{array}{l}2001 \\
\text { (II) }\end{array}$ & 2001: 7909 & National & Yes & Unknown & $\begin{array}{l}\text { 2001: } 28 \\
\text { 2005: } 22\end{array}$ & N/A & $\mathrm{N} / \mathrm{A}$ & $\begin{array}{l}\text { \over } 4 \\
\text { yrs }\end{array}$ \\
\hline & & $\begin{array}{l}2005 \\
(I I I)\end{array}$ & 2005: 7695 & & & & & & & \\
\hline $\begin{array}{l}\text { 11. Laos } \\
\text { People's } \\
\text { Democratic } \\
\text { Republic } \\
\end{array}$ & $\begin{array}{l}\text { World Health Survey } \\
\text { (IPAQ) [16] }\end{array}$ & 2003 & 4640 & National & Yes & all & 88 & $\begin{array}{l}143 \\
\text { median }\end{array}$ & N/A & N/A \\
\hline \multirow[t]{2}{*}{ 12. Malaysia } & $\begin{array}{l}\text { WHO STEPS Survey } \\
\text { (GPAQ) [46] }\end{array}$ & 2005 & 3040 & National & Yes & $W, T, L$ & 40 & $\begin{array}{l}189 \\
\text { median }\end{array}$ & N/A & $\mathrm{N} / \mathrm{A}$ \\
\hline & $\begin{array}{l}\text { World Health Survey } \\
\text { (IPAQ) [16] }\end{array}$ & 2003 & 5563 & National & Yes & all & 54 & $\begin{array}{l}446 \\
\text { median }\end{array}$ & & \\
\hline
\end{tabular}


Table 1 Prevalence of physical activity in the Asia-Pacific region (Continued)

\begin{tabular}{|c|c|c|c|c|c|c|c|c|c|c|}
\hline 13. Maldives & $\begin{array}{l}\text { WHO STEPS Survey } \\
\text { (GPAQ) [47] }\end{array}$ & 2004 & 2000 & $\begin{array}{l}\text { Sub- } \\
\text { national }\end{array}$ & Yes & W & 7 & N/A & N/A & $\mathrm{N} / \mathrm{A}$ \\
\hline \multirow[t]{3}{*}{$\begin{array}{l}\text { 14. Marshall } \\
\text { Islands }\end{array}$} & $\begin{array}{l}\text { WHO NCD STEPS } \\
\text { Survey (GPAQ) [48] }\end{array}$ & 2002 & 3045 & National & Yes & $W, T, L$ & 34 & $\begin{array}{l}\text { W: } 18 \\
\text { mean }\end{array}$ & $\mathrm{N} / \mathrm{A}$ & $\mathrm{N} / \mathrm{A}$ \\
\hline & & & & & & & & $\begin{array}{l}\mathrm{T}: 45 \\
\text { mean } \\
\end{array}$ & & \\
\hline & & & & & & & & $\begin{array}{l}\mathrm{L}: 12 \\
\text { mean }\end{array}$ & & \\
\hline $\begin{array}{l}15 . \\
\text { Micronesia } \\
\text { (Federated } \\
\text { States of) }\end{array}$ & $\begin{array}{l}\text { WHO NCD STEPS } \\
\text { Survey (GPAQ) [49] }\end{array}$ & 2005 & 1638 & $\begin{array}{l}\text { Sub- } \\
\text { national }\end{array}$ & Yes & $W, T, L$ & 36 & 77 mean & N/A & $N / A$ \\
\hline $\begin{array}{l}16 . \\
\text { Mongolia }\end{array}$ & $\begin{array}{l}\text { WHO NCD STEPS } \\
\text { Survey (GPAQ) [50] }\end{array}$ & 2005 & 3411 & National & Yes & $W, T, L$ & 89 & $\begin{array}{l}189 \\
\text { median }\end{array}$ & $\mathrm{N} / \mathrm{A}$ & N/A \\
\hline \multirow[t]{3}{*}{$\begin{array}{l}17 . \\
\text { Myanmar }\end{array}$} & $\begin{array}{l}\text { WHO STEPS Survey } \\
\text { (GPAQ) [51] }\end{array}$ & 2003 & 2163 & $\begin{array}{l}\text { Sub- } \\
\text { national } \\
\text { (rural) }\end{array}$ & Yes & $W, T, L$ & 65 & $\begin{array}{l}918 \\
\text { median }\end{array}$ & N/A & $N / A$ \\
\hline & $\begin{array}{l}\text { WHO STEPS Survey } \\
\text { (GPAQ) [52] }\end{array}$ & & 2285 & $\begin{array}{l}\text { Sub- } \\
\text { national } \\
\text { (urban) }\end{array}$ & Yes & $W, T, L$ & 51 & $\begin{array}{l}288 \\
\text { median }\end{array}$ & & \\
\hline & $\begin{array}{l}\text { World Health Survey } \\
\text { (IPAQ) [16] }\end{array}$ & & 5517 & National & Yes & all & 91 & $\begin{array}{l}694 \\
\text { median }\end{array}$ & & \\
\hline 18. Nauru & $\begin{array}{l}\text { WHO NCD STEPS } \\
\text { Survey (GPAQ) [53] }\end{array}$ & 2004 & 2272 & National & Yes & $W, T, L$ & N/A & $\begin{array}{l}203 \\
\text { median }\end{array}$ & $\begin{array}{l}17 \% \text { physically } \\
\text { inactive = no PA }\end{array}$ & $N / A$ \\
\hline \multirow[t]{6}{*}{ 19. Nepal } & $\begin{array}{l}\text { WHO STEPS Survey } \\
\text { (GPAQ) [54] }\end{array}$ & 2003 & 2030 & $\begin{array}{l}\text { Sub- } \\
\text { national }\end{array}$ & Yes & $W, T, L$ & 18 & N/A & \% Inactive: & $\mathrm{N} / \mathrm{A}$ \\
\hline & & & & & & & & & W: 51 & \\
\hline & & & & & & & & & T: 19 & \\
\hline & & & & & & & & & L: 86 & \\
\hline & $\begin{array}{l}\text { WHO STEPS Survey } \\
\text { (GPAQ) [55] }\end{array}$ & $\begin{array}{l}2004- \\
5\end{array}$ & 7792 & $\begin{array}{l}\text { Sub- } \\
\text { national }\end{array}$ & Yes & $W, T, L$ & $N / A$ & $\begin{array}{l}450 \\
\text { median }\end{array}$ & & \\
\hline & $\begin{array}{l}\text { World Health Survey } \\
\text { (IPAQ) [16] }\end{array}$ & 2003 & 7945 & National & Yes & all & 92 & $\begin{array}{l}1351 \\
\text { median }\end{array}$ & & \\
\hline \multirow[t]{6}{*}{$\begin{array}{l}\text { 20. New } \\
\text { Zealand }\end{array}$} & $\begin{array}{l}\text { Sport and Physical } \\
\text { Activity Survey [56] }\end{array}$ & $\begin{array}{l}1997 / \\
98 \\
\end{array}$ & 12500 & National & Unknown & L & 1997/98: 67 & N/A & $\mathrm{N} / \mathrm{A}$ & $\begin{array}{l}\text { 个over } 3 \\
\text { yrs }\end{array}$ \\
\hline & & $\begin{array}{l}1998 / \\
99 \\
\end{array}$ & & & & & 1998/99: 68 & & & \\
\hline & & $\begin{array}{l}2000 / \\
01\end{array}$ & & & & & 2000/01: 70 & & & \\
\hline & $\begin{array}{l}\text { New Zealand Health } \\
\text { Survey }[57,58]\end{array}$ & $\begin{array}{l}2002- \\
3\end{array}$ & $\begin{array}{l}\text { 2002-3: } \\
12929\end{array}$ & & Yes & $\mathrm{L}$ & 2002-3: 52 & & & $\begin{array}{l}\leftrightarrow \text { over } \\
4 \text { yrs }\end{array}$ \\
\hline & & $\begin{array}{l}2006- \\
7\end{array}$ & $\begin{array}{l}2006-7: \\
12488\end{array}$ & & & & 2006-7: 51 & & & \\
\hline & $\begin{array}{l}\text { IPAQ Short Form } \\
{[17]}\end{array}$ & 2003 & 1495 & & Yes & all & 88 & & & $\overline{N / A}$ \\
\hline 21. Pakistan & $\begin{array}{l}\text { World Health Survey } \\
\text { (IPAQ) [16] }\end{array}$ & 2003 & 5610 & National & Yes & All & 80 & $\begin{array}{l}623 \\
\text { median }\end{array}$ & $\mathrm{N} / \mathrm{A}$ & $\mathrm{N} / \mathrm{A}$ \\
\hline $\begin{array}{l}22 . \\
\text { Philippines }\end{array}$ & $\begin{array}{l}\text { World Health Survey } \\
\text { (IPAQ) [16] }\end{array}$ & 2003 & 9535 & National & Yes & All & 93 & $\begin{array}{l}1158 \\
\text { median }\end{array}$ & $\mathrm{N} / \mathrm{A}$ & $\mathrm{N} / \mathrm{A}$ \\
\hline \multirow[t]{3}{*}{ 23. Samoa } & $\begin{array}{l}\text { WHO NCD STEPS } \\
\text { Survey (GPAQ) [59] }\end{array}$ & 2002 & 2817 & National & Yes & $W, T, L$ & 50 & W: 9 mean & $\mathrm{N} / \mathrm{A}$ & $\mathrm{N} / \mathrm{A}$ \\
\hline & & & & & & & & $\mathrm{T}: 3$ mean & & \\
\hline & & & & & & & & L: 2 mean & & \\
\hline \multirow[t]{2}{*}{$\begin{array}{l}24 . \\
\text { Singapore }\end{array}$} & $\begin{array}{l}\text { Singapore National } \\
\text { Health Survey }[60,61]\end{array}$ & 1992 & 1992: 3568 & National & Unknown & L & 1992: 14 & $\mathrm{~N} / \mathrm{A}$ & $\mathrm{N} / \mathrm{A}$ & $\begin{array}{l}\text { 个over } \\
12 \text { yrs }\end{array}$ \\
\hline & & 1998 & 1998: 4723 & & & & 1998: 17 & & & \\
\hline
\end{tabular}


Table 1 Prevalence of physical activity in the Asia-Pacific region (Continued)

\begin{tabular}{|c|c|c|c|c|c|c|c|c|c|c|}
\hline & & 2004 & 2004: 4084 & & & & 2004: 25 & & & \\
\hline \multirow[t]{2}{*}{$\begin{array}{l}\text { 25. Sri } \\
\text { Lanka }\end{array}$} & $\begin{array}{l}\text { WHO NCD STEPS } \\
\text { Survey (GPAQ) [62] }\end{array}$ & 2003 & 3,000 & $\begin{array}{l}\text { Sub- } \\
\text { national }\end{array}$ & Yes & $W, L$ & 85 & N/A & $\begin{array}{l}\text { Mean mins spent } \\
\text { in PA/day: } 257\end{array}$ & $\mathrm{~N} / \mathrm{A}$ \\
\hline & $\begin{array}{l}\text { World Health Survey } \\
\text { (IPAQ) [16] }\end{array}$ & $\begin{array}{l}2002- \\
3 \\
\end{array}$ & 5,464 & National & Yes & All & 90 & $\begin{array}{l}1,089 \\
\text { median }\end{array}$ & & \\
\hline \multirow[t]{5}{*}{ 26. Taiwan } & $\begin{array}{l}\text { National Health } \\
\text { Survey of Taiwan } \\
{[63-66]}\end{array}$ & 2001 & 15,559 & National & unknown & $\bar{L}$ & (1) 21 & $N / A$ & (1) $N / A$ & (1) $N / A$ \\
\hline & $\begin{array}{l}\text { National Council on } \\
\text { Physical Fitness and } \\
\text { Sports }[63,64]\end{array}$ & 2000 & 2000: 1021 & & & $\mathrm{~L}$ & (2) $N / A$ & & $\begin{array}{l}(2)>3 \times w k,>30 \\
\text { mins }\end{array}$ & $\begin{array}{l}\text { (2) } \\
\uparrow \text { over } 3 \\
\text { yrs }\end{array}$ \\
\hline & & 2001 & 2001: 8573 & & & & & & 2001: 10\% & \\
\hline & & 2004 & 2004: 4073 & & & & & & 2004: 14\% & \\
\hline & $\begin{array}{l}\text { IPAQ Short Form } \\
{[17]}\end{array}$ & 2004 & 4846 & & $\overline{Y e s}$ & all & (3) 58 & & (3) $\mathrm{N} / \mathrm{A}$ & (3) $N / A$ \\
\hline \multirow[t]{4}{*}{ 27. Thailand } & $\begin{array}{l}\text { Adapted IPAQ (long } \\
\text { form) (2003) IPAQ } \\
\text { short version (2004- } \\
\text { 6) [65] }\end{array}$ & 2003 & $\begin{array}{l}\text { 2003: } \\
11462\end{array}$ & National & unknown & all & 2003: 59 & N/A & $\mathrm{N} / \mathrm{A}$ & $\begin{array}{l}\text { ^over } 3 \\
\text { yrs }\end{array}$ \\
\hline & & 2004 & 2004: 8383 & & & & 2004: 79 & & & \\
\hline & & 2006 & $\begin{array}{l}2006: \\
15158\end{array}$ & & & & 2005: 83 & & & \\
\hline & & & & & & & 2006: 79 & & & \\
\hline 28. Tokelau & $\begin{array}{l}\text { WHO NCD STEPS } \\
\text { Survey (GPAQ) [66] }\end{array}$ & 2005 & 586 & National & Yes & $W, T, L$ & 58 & 111 mean & N/A & N/A \\
\hline \multirow[t]{2}{*}{ 29. Vietnam } & $\begin{array}{l}\text { WHO NCD STEPS } \\
\text { Survey (GPAQ) [7] }\end{array}$ & 2005 & 1906 & $\begin{array}{l}\text { Sub- } \\
\text { national }\end{array}$ & Yes & $W, T, L$ & 56 & N/A & N/A & N/A \\
\hline & $\begin{array}{l}\text { World Health Survey } \\
\text { (IPAQ) [16] }\end{array}$ & $\begin{array}{l}2002- \\
3\end{array}$ & 3009 & National & Yes & All & 92 & 98 median & & \\
\hline
\end{tabular}

WHO NCD STEPS WHO STEPwise approach to Non-Communicable Disease surveillance, InterASIA International Collaborative Study of Cardiovascular Disease in Asia, GPAQ Global Physical Activity Questionnaire, IPAQ International Physical Activity Questionnaire, $W$ Physical activity in the 'work' domain, $T$ Physical activity in the 'travel' domain, L Physical activity in the 'leisure' domain, $\uparrow$ Increase, $\leftrightarrow$ No substantial change, $\downarrow$ Decrease, N/A Not applicable, II Second survey version, III Third survey version

information using several instruments including IPAQ, GPAQ and other leisure-time physical activity surveys. It highlights the large variation between estimates obtained using different measurement tools, and thus demonstrates the need for standardisation of samples and of measures.

This review has demonstrated that physical activity estimates vary widely, even within a single country using different surveys over similar time periods. For example, with the exception of India and Sri Lanka, 11 of the 13 countries with more than one survey reporting the percentage sufficiently active give substantially different estimates from different surveys. Similarly, all four countries reporting values for median MET-minutes from different surveys showed substantial variation in reported physical activity.

Notably, household and garden activities are included in GPAQ but for the remainder of surveys using alternative instruments, physical activity in this domain was unknown. Consequently, there may be an underestimation of overall activity level. This is particularly relevant to developing countries in the Asia-Pacific region, where this domain may contribute a higher proportion to total than in more industrialised countries. Careful consideration should be given to the need to measure physical activity in different domains, and the consequences of these methodological decisions on prevalence estimates.

In developing countries outside the Asia-Pacific region, a similar situation regarding physical activity surveillance measures is evident. In two Brazilian studies which both used the multi-domain IPAQ long-form questionnaire, the prevalence of inactive adults was reported as $41.1 \%$ in a Brazilian city survey [67] and $26.1 \%$ inactive in a national sample [14]. A third Brazilian study [68] reports that only $3 \%$ of adults were sufficiently active in leisure-time alone, compared with the higher estimates derived from using the more generic IPAQ measure. Similarly, in Saudi Arabia, one estimate [69] gives high inactivity prevalence of $96 \%$ in a largescale national survey examining leisure-time activity, yet another [8] reports only $41 \%$ inactive using IPAQ in over 1000 adults living in the capital city. These studies 


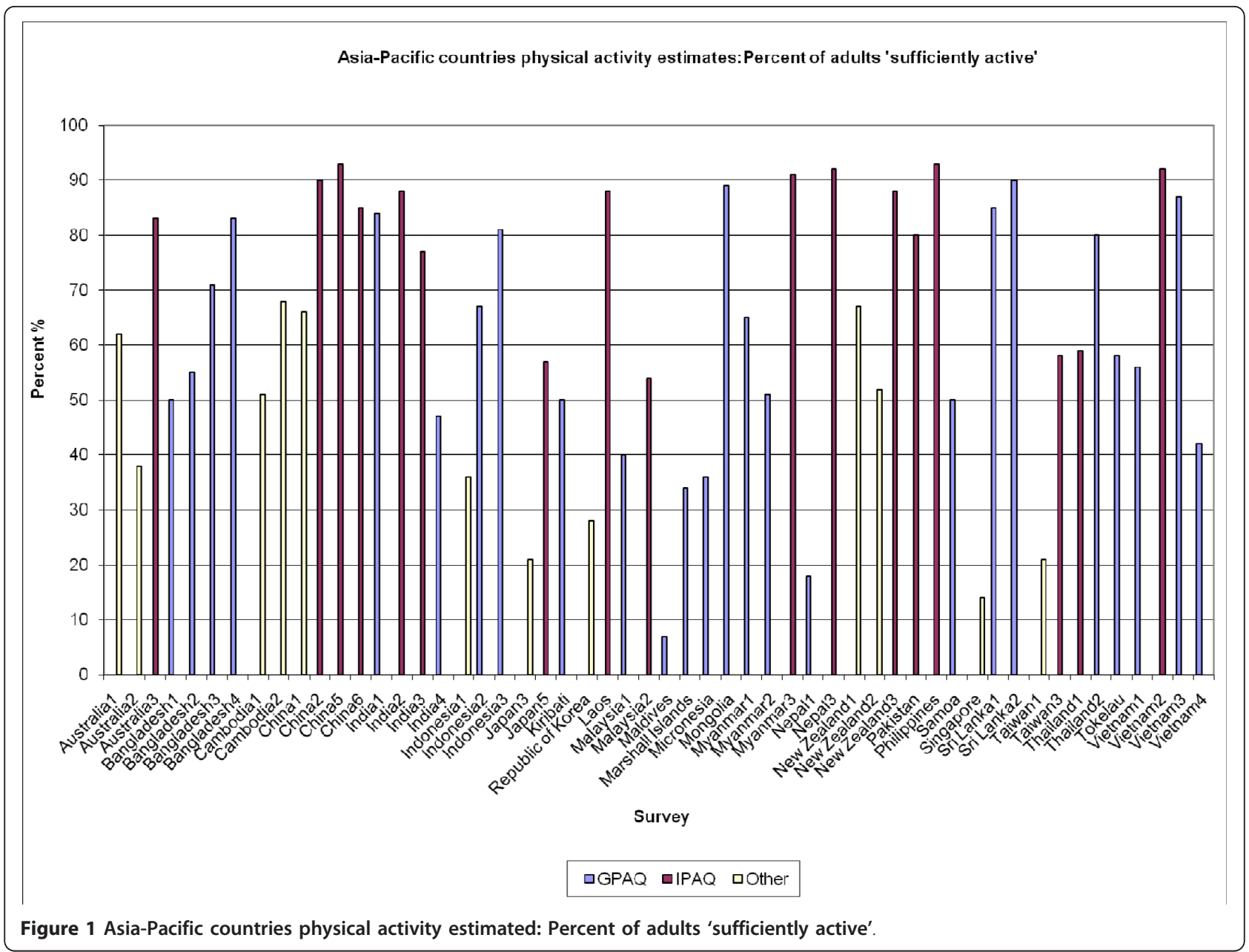

confirm the large prevalence differences between total physical activity measures and leisure time-only surveys.

Physical activity is an important indicator for the health and sport sectors, and also for other sectors such as transport and urban planning. This is the first time physical activity estimates have been collated among adult populations, especially in LMIC. The policy and practical implications of this work are profound, particularly in the setting of burgeoning obesity prevalence and the NCD epidemic confronting many Asia-Pacific nations. Accurate and consistent measurement of physical activity is essential to guide evidence-based public health practice and facilitate and target health promotion initiatives. Moreover, accurately documenting participation in various domains of physical activity may facilitate a multi-sectoral 'health in all policies' approach.

Whilst our criteria for selection of studies were carefully considered, the use of grey literature can be problematic. The true denominator of actual studies cannot be ascertained, so this is a collection of estimates. Thorough review and contact with national agencies led us to consider this a comprehensive distillation, but the epithet 'systematic review' cannot be attached to these methods. Additionally, reliability and validity of instruments reported in the grey literature is unknown, potentially limiting their accuracy. Nonetheless, since most of the international physical activity measures from LMIC are reported in the grey literature, this is a large collection which identifies important issues in physical activity surveillance and estimation, within and between countries. It also identifies problems that might be caused by incompatible surveillance systems. Whilst some of the variation seen in this review reflects real differences in physical activity, other factors such as the domains of activity included in the survey questionnaire and population sampling differences, may have influenced these results. Even using the same survey and protocol, countries similar in size, culture or stage of development may show unexplained differences in prevalence [16]. Assessing reliability and validity of existing measures in the specific population where measurement is intended is advised. More intensive research, using a mix of 
qualitative and quantitative methodologies, may also be needed to understand and explain observed differences, prior to further surveillance.

Included in this research agenda is population-level physical activity monitoring in the countries listed in Additional file 2 where data was unavailable or unknown for the period 2000-2010, as well as the need for sub-samples to be compared with objective physical activity measures, using accelerometry or pedometers at the population level [70-72]. These objective studies point to over-estimation of self-report physical activity levels, which may mean that current burden of disease calculations are under-estimating the population attributable fraction for inactivity. Nonetheless, even in the absence of objective surveillance, physical activity prevalence across the Asia-Pacific region should be compared using identical sampling methods across surveys and using established, reliable and valid measures.

\section{Conclusions}

There is an urgent need for standardization of physical activity measures and survey methods used within and between countries, in order to accurately document risk factor prevalence and population trends. Between-country comparisons are also easier when methodologically comparable data collection techniques are adopted, and when identical protocols and analytic methods are used. It is encouraging that many developing countries collect physical activity survey data, either as part of NCD surveillance or from other sectors, and report on population participation rates, active transport, or total physical activity. This review highlights differences between estimates and demonstrates that use of different questionnaires, even within the same country, may yield discrepant results. The challenge in public health surveillance is to work towards comparability of measures and methods to assess physical activity in populations.

\section{Additional material}

Additional file 1: Search Terms Used In Medline Search For Asia

Pacific Physical Activity Data

Additional file 2: Countries where national or representative data was unavailable or unknown for the period 2000-2010.

\section{Acknowledgements}

We would like to thank the Asia Pacific countries that provided information for this review.

\section{Author details}

${ }^{1}$ Prevention Research Collaboration, School of Public Health, University of Sydney, Sydney, Australia. ${ }^{2}$ Greater Green Triangle University Department of Rural Health, Flinders and Deakin Universities, Warrnambool, Australia.

\section{Authors' contributions}

RM and MA participated in data collection and synthesis. $A B$ conceived of the study and participated in its design. All authors helped draft and approved the final manuscript.

\section{Competing interests}

The authors declare that they have no competing interests.

Received: 26 August 2011 Accepted: 17 January 2012

Published: 17 January 2012

\section{References}

1. Haskell WL, Lee IM, Pate RR, Powell KE, Blair SN, Franklin BA, Macera CA, Heath GW, Thompson PD, Bauman A: Physical activity and public health: updated recommendation for adults from the American college of sports medicine and the American heart association. Med Sci Sports Exerc 2007, 39(8):1423-1434.

2. World Health Organisation: Preventing Chronic Disease-a vital investment WHO Geneva: World Health Organisation; 2005.

3. World Health Organisation: Global health risks: mortality and burden of disease attributable to selected major risks Geneva: World Health Organisation; 2009.

4. Dietz WH: The role of lifestyle in health: the epidemiology and consequences of inactivity. Proc Nutr Soc 1996, 55(3):829-840.

5. Warburton DER, Nicol CW, Bredin SSD: Health benefits of physical activity: the evidence. CMAJ 2006, 174(6):801-809.

6. Armstrong T, Bauman A, Davies J: Physical activity patterns of Australian adults Canberra: Australian Institute of Health and Welfare; 2000.

7. Trinh OT, Nguyen ND, Dibley MJ, Phongsavan P, Bauman AE: The prevalence and correlates of physical inactivity among adults in Ho Chi Minh City. BMC Publ Health 2008, 8:204

8. Hallal PC, Victoria CG, Wells JCK, Lima RC: Physical inactivity: prevalence and associated variables in Brazilian adults. Med Sci Sports Exerc 2003, 35(11):1894-1900.

9. Bauman A, Phongsavan P, Schoeppe S, Owen N: Physical activity measurement-a primer for health promotion. Promot Educ 2006, 13(2):92-103.

10. World Health Organisation: Global recommendations on physical activity for health Geneva: World Health Organisation; 2010.

11. Centers for Disease Control and Prevention (CDC): Behavioural Risk Factor Surveillance System State Questionnaire Atlanta: Centers for Disease Control and Prevention; 2003.

12. Craig CL, Russell SJ, Cameron C, Bauman A: Twenty year trends in physical activity among Canadian adults. Can J Public Health 2004, 95(1):59-63.

13. Prättälä R, Helasoja V: The Finbalt Health Monitor: Monitoring health behaviour in Finland and the Baltic countries. In Global Behaviour Risk Factor Surveillance. Edited by: McQueen D, Puska P. New York: Kluwer; 2003:57-72.

14. Craig $C L$, Marshall $A L$, Sjöström M, Bauman A, Booth ML, Ainsworth BE, Pratt M, Ekelund U, Yngve A, Sallis JF, Oja P: International physical activity questionnaire: 12-country reliability and validity. Med Sci Sports Exerc 2003, 35(8):1381-1395.

15. Armstrong T, Bull F: Development of the world health organization global physical activity questionnaire (GPAQ). J Public Health 2006, 14:66-70.

16. Guthold R, Ono T, Strong KL, Chatterji S, Morabia A: Worldwide variability in physical inactivity: A 51 country survey. Am J Prev Med 2008, 34(6):486-494

17. Bauman A, Bull F, Chey T, Craig C, Ainsworth BE, Sallis JF, Bowles HR, Hagstromer M, Sjostrom M, Pratt M, Ips Group: The international prevalence study on physical activity: results from 20 countries. Int $J$ Behav Nutr Phys Act 2009, 6(1):21.

18. World Health Organisation Global Infobase:, Available online at www.who. int/infobase [Accessed September 2010].

19. Bull FC, Maslin TS, Armstrong T: Global physical activity questionnaire (GPAQ): nine country reliability and validity study. J Phys Act Health 2009, 6(6):790-804.

20. The World Bank Atlas Method: The World Bank., Available online at http:// data.worldbank.org/about/country-classifications/world-bank-atlas-method [Accessed September 2010]. 
21. World Health Organization for the Western Pacific Region (WPRO): Western Pacific Regional Action Plan for Noncommunicable Diseases: A Region Free of Avoidable NCD Deaths and Disability Manila: World Health Organization; 2009.

22. World Health Organization: The world health report 2002-Reducing Risks, Promoting Healthy Life Geneva: World Health Organization; 2002.

23. Asia-Pacific Physical Activity Network (AP-PAN): 2010, Available online at www.ap-pan.org [Accessed September 2010].

24. Ainsworth BE, Haskell WL, Whitt MC, Irwin ML, Swartz AM, Strath SJ, O'Brien WL, Bassett DR, Schmitz KH, Emplaincourt PO, Jacobs DR, Leon AS: Compendium of physical activities: an update of activity codes and MET intensities. Med Sci Sports Exerc 2000, 32(9 Suppl):S498-S504.

25. Bauman A, Bellew B, Vita P, Brown W, Owen N: Getting Australia active: towards better practice for the promotion of physical activity Melbourne: National Public Health Partnership; 2002.

26. Australian Institute for Health and Welfare (AlHW): Physical activity, diet and body weight: results from the 2001 National Health Survey Canberra: AlHW; 2004.

27. Merom D, Phongsavan $P$, Chey T, Bauman A: Long term changes in leisure time walking, moderate and vigorous exercise: were they influenced by the national physical activity guidelines? I Sci Med Sport 2006, 9(3):199-208.

28. Merom D: Personal communication (extension of Merom et al. 2006, including most recent survey). 2009.

29. Rahman M, Meerjady SF, Akter SFU, Hossain S, Mascie-Taylor CGN: Behavioural risk factors of non-communicable diseases in Bangladesh Tobacco Usage, dietary pattern and physical activity status (STEPS Survey) Geneva: World Health Organisation; 2002, [www.who.int/chp/steps/ BangladeshSTEPSReport.pdf] [Accessed September 2010].

30. King H, Keuky L, Seng S, Khun T, Roglic G, Pinget M: Diabetes and associated disorders in Cambodia: two epidemiological surveys. Lancet 2005, 366(9497):1633-1639.

31. Mutner P, Donfeng G, Wildman RP, Chen J, Qan W, Whelton PK, He J: Prevalence of physical activity among Chinese adults: Results of the international collaborative study of cardiovascular disease in Asia. Am J Public Health 2005, 95(9):1631-1636.

32. Fu FH, Fung L: The cardiovascular health of residents in selected metropolitan cities in China. Prev Med 2004, 38(4):458-467.

33. Shi J, Liu M, Zhang Q, Lu M, Quan H: Male and female adult population health status in China: a cross-sectional national survey. BMC Publ Health 2008, 8:277.

34. Cornelius M, Decourten M, Pryor J, Saketa S, Waganivalu TK, Laqeretabua A, Chung E: Fiij Non-Communicable Diseases (NCD) Steps Survey 2002 Geneva: Ministry of Health; World Health Organisation; Fiji School of Medicine; Menzies Centre for Public Health Research, University of Tasmania; 2002, [www.who.int/chp/steps/FijiSTEPSReport.pdf] [Accessed September 2010].

35. Shah B: Development of sentinel health monitoring centres for surveillance of risk factors of non-communicable diseases in India Geneva: World Health Organisation; 2005, [www.who.int/chp/steps/IndiaSTEPSReport_6Centers.pdf] [Accessed September 2010].

36. Soemantri S, Pradono J, Hapsari D: SURKESNAS 2001. National Household Health Survey Morbidity Study. NCD Risk Factors in Indonesia, 2001 Geneva: World Health Organisation; 2001, [www.who.int/chp/steps/ STEPS_Report_Indonesia_National_2001.pdf] [Accessed September 2010].

37. Centre for Disease Control Research and Development; NIHRD Ministry of Health Republic of Indonesia; World Health Organisation: Final Report. Integrated Community Based Intervention on Major NCDs In Depok Municipality Geneva: World Health Organisation; 2003, [www.who.int/chp/ steps/IndonesiaSTEPSReport2003.PDF] [Accessed September 2010].

38. Centre for Disease Control Research and Development; NIHRD Ministry of Health Republic of Indonesia; World Health Organisation: Monitoring and Evaluation of the Integrated Community Based Intervention for the Prevention of NCD in Depok, West Java, Indonesia Geneva: World Health Organisation; 2006, [www.who.int/chp/steps/STEPS_Report_Indonesia_Depok_2006.pdf] [Accessed September 2010].

39. Sasakawa Sports Foundation: The 2000 SSF National Sport Life Survey, Executive Summary Tokyo: Sasakawa Sports Foundation; 2001.

40. Sasakawa Sports Foundation: The 2002 SSF National Sport Life Survey, Executive Summary Tokyo: Sasakawa Sports Foundation; 2003.

41. Sasakawa Sports Foundation: The 2004 SSF National Sport Life Survey, Executive Summary Tokyo: Sasakawa Sports Foundation; 2005.
42. Sasakawa Sports Foundation: The 2006 SSF National Sport Life Survey, Executive Summary Tokyo: Sasakawa Sports Foundation; 2007.

43. Ministry of Health: Kiribati NCD Risk Factors STEPS Report Suva: Ministry of Health Kiribati; 2009.

44. Nam JJ, Koh MJ, Choi JS, Kim JH, Lee YH: Korea National Health and Nutrition Examination Survey (KNHANES II) 2001 Geneva: World Health Organisation; 2004

45. Kang E, Choi EJ, Song HJ, Ryou KC, Kim NY, Kim KN, Nam JJ, Park MH, Lee NH: Korea National Health and Nutrition Examination Survey (KNHANES III) 2005, Health behaviour of Adults Geneva: World Health Organisation; 2005.

46. Disease Control Division: Malaysia NCD Surveillance 2006: NCD Risk Factors in Malaysia Kuala Lumpur: Ministry of Health; 2006.

47. Health Information and Research Section: Ministry of Health: Survey on Non Communicable Disease Risk Factors Maldives 2004 Geneva: World Health Organisation; 2004, [www.who.int/chp/steps/MaldivesSTEPSReport2004.pdf] [Accessed September 2010].

48. Ministry of Health: NCD Risk Factors STEPS Report Suva: Ministry of Health Republic of the Marshall Islands; 2007.

49. Department of Health and Social Affairs, Government of the Federal States of Micronesia: NCD Risk Factors STEPS Report, Department of Health and Social Affairs, Government of the Federal States of Micronesia. 2008.

50. World Health Organisation: Mongolian STEPS survey on the prevalence of non-communicable disease risk factors 2006 Geneva: World Health Organisation; 2006, [www.who.int/chp/steps/27\%20December\%202006\% 20Mongolia\%20STEPS\%20Survey.pdf] [Accessed September 2010].

51. World Health Organisation: WHO STEPwise approach to NCD surveillance Myanmar. Disaggregation of urban and rural data (rural) 2003 Geneva: World Health Organisation; 2003, [www.who.int/chp/steps/ MyanmarSTEPSReport2004RURAL.pdf] [Accessed September 2010].

52. World Health Organisation: WHO STEPwise approach to NCD surveillance Myanmar. Disaggregation of urban and rural data (urban) 2003 Geneva: World Health Organisation; 2003, [www.who.int/chp/steps/ MyanmarSTEPSReport2004URBAN.pdf] [Accessed October 2008].

53. Keke K, Phongsavan P, Bacigaluno DL, Smith B, Thoma R, Riley L, Waidubu G, Galea G, Pryor J, Rai S, Chey T: Nauru NCD Risk Factors. STEPS report 2005 Suva: World Health Organisation; 2007, [www.who.int/chp/ steps/Printed_STEPS_Report_Nauru.pdf] [Accessed September 2010].

54. World Health Organisation: Research report on NCD risk factor surveillance in Nepal, 2003 Geneva: World Health Organisation; 2003, [www.who.int/chp/ steps/NepalSTEPSReport2003Kathmandu.pdf] [Accessed September 2010].

55. World Health Organisation: Surveillance of risk factors for non-communicable diseases in Nepal. Report of survey in Ilam, Lalitpur and Tanahu Geneva: World Health Organisation; 2006, [www.who.int/chp/steps/ NepalSTEPSReport2005_3districts.pdf] [Accessed October 2008].

56. New Zealand Government: SPARC trends: trends in participation in sport and active leisure, 1997-2001 Wellington: New Zealand Government; 2003, [http://www.activenzsurvey.org.nz] [Accessed September 2010].

57. Ministry of Health: A Portrait of Health: Key results of the 2006/07 New Zealand Health Survey Wellington: Ministry of Health; 2008.

58. Ministry of Health: A portrait of Health: Key results of the 2002/03 New Zealand Health Survey Wellington: Ministry of Health; 2004.

59. Samoa Ministry of Health, World Health Organisation, Department of Epidemiology and Preventative Medicine, Monash University: Samoa NCD Risk Factors. STEPS Report Suva: World Health Organisation; 2007.

60. Ministry of Health Singapore: National Health Survey. 2004, Personal communication.

61. Statistics Singapore: National Health Survey 2004 Singapore: Statistics Singapore; 2005, [www.singstat.gov.sg/pubn/papers/people/ssnsep05-pg1920.pdf] [Accessed September 2010].

62. Somatunga LC: NCD Risk factor survey in Sri Lanka (STEP Survey) Geneva: World Health Organisation; 2004, [www.who.int/chp/steps/ SriLankaSTEPSReport2003.pdf] [Accessed September 2010].

63. Ku PW, Fox KR, McKenna J, Peng TL: Prevalence of leisure time physical activity in Taiwanese adults: results of four national surveys, 2000-2004. Prev Med 2006, 43(6):454-457.

64. Lin YC, Wen CP, Wai JPM: Leisure-time physical activity and its association with health behaviors, health status and health related quality of life among Taiwanese adults. J Public Health 2007, 26:218-228.

65. Ministry of Health: Thailand: Personal Communication; 2007. 
66. World Health Organisation: Tokelau NCD Risk Factors. STEPS Report, 2004 Suva: World Health Organisation; 2007, [www.who.int/chp/steps/ STEPS_Report_Tokelau.pdf] [Accessed September 2010].

67. Monteiro CA, Conde WL, Matsudo SM, Matsudo VR, Bonsenor IM, Lotufo PA: A descriptive epidemiology of leisure-time physical activity in Brazil, 1996-1997. Rev Panam Salud Publica 2003, 14(4):246-254.

68. Al-Nozha MM, Al-Hazzaa HM, Arafah MR, Al-Khandra A, Al-Mazrou YY, AlMaatoug MA, Khan NB, Al-Marzouki K, Al-Harthi SS, Abdullah M, AlShahid MS: Prevalence of physical activity and inactivity among Saudis aged 30-70 years. A population-based cross-sectional study Saudi Med J 2007, 28(4):559-568.

69. Al-Hazzaa HM: Health-enhancing physical activity among Saudi adults using the international physical activity questionnaire (IPAQ). Public Health Nutr 2007, 10(1):59-64.

70. Atienza AA, Moser RP, Perna F, Dodd K, Ballard-Barbash R, Troiano RP, Berrigan D: Self-reported and objectively-measured activity related to biomarkers using NHANES. Med Sci Sports Exerc 2011, 43(5):815-821.

71. Hagströmer M, Troiano RP, Siöström M, Berrigan D: Levels and patterns of objectively assessed physical activity-a comparison between Sweden and the United States. Am J Epidemio/ 2010, 171(10):1055-1064.

72. Craig CL, Cameron C, Griffiths JM, Tudor-Locke C: Descriptive epidemiology of youth pedometer-determined physical activity: CANPLAY. Med Sci Sports Exerc 2010, 42(9):1639-1643.

\section{Pre-publication history}

The pre-publication history for this paper can be accessed here: http://www.biomedcentral.com/1471-2458/12/41/prepub

doi:10.1186/1471-2458-12-41

Cite this article as: Macniven et al:: A review of population-based prevalence studies of physical activity in adults in the Asia-Pacific region. BMC Public Health 2012 12:41.

\section{Submit your next manuscript to BioMed Central} and take full advantage of:

- Convenient online submission

- Thorough peer review

- No space constraints or color figure charges

- Immediate publication on acceptance

- Inclusion in PubMed, CAS, Scopus and Google Scholar

- Research which is freely available for redistribution

Submit your manuscript at www.biomedcentral.com/submit 